\title{
Under the spell of SARS-CoV-2: A closer look at the sociopolitical dynamics
}

\author{
Neha Khetrapal $^{1} \cdot$ Gunjan Khera $^{2}$
}

Received: 14 August 2020 / Accepted: 5 May 2021 / Published online: 12 May 2021

(C) Springer Nature Switzerland AG 2021

\begin{abstract}
Sitting on the fine line between pathogen 'transmissibility' and 'severity', the Behavioural Immune System (BIS) is responsible for activating behaviours that minimise infection risks and maximise fitness. To achieve self-preservation, the BIS also fuels social and political attitudes. We aim to explain societal changes that may be sparked by COVID-19 by highlighting links between human evolutionary history and our psychological faculties mediated by the BIS.
\end{abstract}

Keywords Social and political changes $\cdot$ Pandemic $\cdot$ Self-preservation · Authoritarianism

\section{Introduction}

Beyond the immediate health risks, the social and political consequences associated with the spread of SARS-CoV-2 are of great importance. ${ }^{1}$ As researchers interested in cross-cultural political psychology, we want to explain the rise of authoritarianism, characterised by hostility towards other social groups and ethnocentrism, across the world in the wake of COVID-19. The aim is to provide an explanation for the global rise of conservative attitudes. Acknowledging that the current situation is but a snapshot in a still evolving crisis, these explanations are intended as an initial

\footnotetext{
1 This note belongs to the Topical Collection, "Seeing Clearly Through COVID-19: Current and Future Questions for the History and Philosophy of the Life Sciences", edited by G. Boniolo and L. Onaga.

Neha Khetrapal

nkhetrapal@jgu.edu.in

1 Jindal Institute of Behavioural Sciences, O.P. Jindal Global University, Sonipat, Haryana, India

2 College of Humanities and Social Sciences, Department of Cognitive Sciences, United Arab

Emirates University, Al Ain, UAE
} 
social scientific cut rather than a complete evaluation of the social consequences of the pandemic.

Compared to the other recent SARS outbreak in 2003, COVID-19 is on a different scale. Thus far, the pandemic has had the effect of strengthening national borders and concomitant anti-foreign sentiments, which the world was already witnessing on account of Brexit and as part of the US-American executive policies. Similar social and political trends were also observed in Asia, where there had been reports of rising nationalism during the first half-year of the pandemic. Given these trends, is it reasonable to ask whether the global population will eventually find itself in a politically and socially closed-off world as a consequence of COVID-19?

\section{Ecological forces and us}

In answering this question, we first highlight the pathogen avoidance responses that humans have evolved in the course of a long evolutionary history. COVID-19 is a rich ground for activating these self-protective responses. Throughout the human evolutionary trajectory, pathogen stress created strong survival pressures culminating in the development of the immune system to ward off diseases (Kasahara, 2011).

The immune system comprises the 'behavioural immune system' (BIS) and the 'physiological immune system' (PIS). The former entails a complex set of evolved cognitive and behavioural mechanisms. These psychological mechanisms work to detect visible signs of infection (e.g., signs of pustules) and accelerate adaptive responses (e.g., avoidance) to pre-empt the negative impact of pathogen stress even before an infection has struck (Schaller \& Park, 2011). While the PIS serves as the first line of defence against an infection, the BIS is activated by 'visible' cues, e.g., specific facial anomalies associated with infections (Ryan et al., 2012). Often, these overt cues do not perfectly predict who carries a disease. As such, the BIS may 'overreact' and lead to false positive errors. Therefore, the activation of the BIS can have far-reaching implications for a host of psychological functions, including political preferences (Billingsley et al., 2018) and social relationships (Ji et al., 2019).

At any rate, the activation of the BIS, including any 'overreaction', is considered adaptive, as failing to detect a potential pathogen cue can have dire consequences (Murray \& Schaller, 2016). The implication is that the PIS takes a 'backseat' in situations where physiological immunity does not yet exist or is likely to be suppressed (see, Miller \& Maner, 2011), which is the case with SARS-CoV-2. In such cases, the BIS takes the 'driving seat' in our self-defence mechanisms.

\section{Extending the ecological explanation}

A radical component of the BIS mechanism involves the exclusion of members of ethnic out-groups. As humans developed an exquisite immune system that is well-equipped to combat pathogens prevalent among the members of a particular social group or in-group, it proved adaptive to avoid any foreign or 'exotic' pathogens carried by out-group members. These evolved behavioural mechanisms, apt 
for pre-industrial, small-scale living conditions, are ill suited for dealing with the infectious threats of the modern world. This is because evolutionary forces lag behind the pace of industrialisation and have failed to fashion pathogen avoidance mechanisms that could be better suited to our contemporary connected world (e.g., Ackerman et al., 2020).

At the group or societal level, pathogens have been found to co-vary globally with authoritarianism and exclusionary attitudes towards ethnic out-group members, because people prefer authoritative leaders to orchestrate collective social action in the service of 'group survival' (refer, Fincher et al., 2008; Gelfand et al., 2011). In line with this theorising, Hartman et al. (2020) documented a direct link between the perceived threat of COVID-19 and authoritarian and anti-immigrant attitudes based on a cross-sectional representative survey in the UK in March 2020. Previous studies show that people in the United States exhibited exclusionary attitudes towards immigrants after simply reading about the health risks associated with swine flu during the HINI pandemic (Huang et al., 2011) and the 2014 Ebola outbreak (Kim et al., 2016).

\section{The proposal}

We invoke these theoretical premises and empirical findings to explain the rise of a pandemic of 'authoritarianism' and 'ethnocentrism' in tandem with COVID-19. The novelty of our proposal lies in the trade-off between virulence and transmissibility of SARS-CoV-2. The either/or relationship of virulence and transmissibility has important implications for the activation of the BIS and the ensuing social and political upheavals.

Viruses tread a fine line between transmissibility and severity (see, Asai \& Nishiura, 2017). Those that are too virulent will incapacitate or even kill infected hosts (e.g., Ebola), which limits their ability to infect others in the long run. Those that are less harmful, on the other hand, will be successful in making copies of themselves and increase the scope of their transmissibility, which is likely to be the pertinent scenario with the impact of SARS-CoV-2 on otherwise 'healthy' hosts. The latter case also serves as an effective ground for the activation of BIS, while the former triggers the PIS.

We therefore argue that the spread of SARS-CoV-2 is likely to be associated with the development of particular socio-political ideologies that hinge on the activation of the BIS for redrawing social boundaries between in- versus outgroups in our complex world. Whether this will lead to novel kinds of 'ethnophobia' or even 'theophobia' remains to be seen. Alternatively, we may witness new cooperation across societies concomitant with a weakening of the 'us' versus 'them' divide; a strategy that requires the inclusion of 'everyone' in a unified global attempt to fight the pathogen. Until the pandemic descends from its current global peak, we hope that this commentary serves as a 'turntable' for speculating 
about its political and social aftermath, and that this will involve the cooperation of researchers from a variety of disciplines.

\section{References}

Ackerman, J. M., Tybur, J. M., \& Blackwell, A. D. (2020). What role does pathogen-avoidance psychology play in pandemics? Trends in Cognitive Sciences, S1364-6613(20), 30280-30281

Asai, Y., \& Nishiura, H. (2017). Joint estimation of the transmissibility and severity of Ebola virus disease in real time. Journal of Biological Systems, 25(04), 587-603

Billingsley, J., Lieberman, D., \& Tybur, J. M. (2018). Sexual disgust trumps pathogen disgust in predicting voter behavior during the 2016 US presidential election. Evolutionary Psychology, 16(2), 1474704918764170

Fincher, C. L., Thornhill, R., Murray, D. R., \& Schaller, M. (2008). Pathogen prevalence predicts human cross-cultural variability in individualism/collectivism. Proceedings of the Royal Society B: Biological Sciences, 275(1640), 1279-1285

Gelfand, M. J., Raver, J. L., Nishii, L., Leslie, L. M., Lun, J., Lim, B. C., et al. (2011). Differences between tight and loose cultures: A 33-nation study. Science, 332, 1100-1104

Hartman, T. K., Stocks, T. V., McKay, R., Gibson-Miller, J., Levita, L., Martinez, A. P., \& Bentall, R. P. (2020). The authoritarian dynamic during the COVID-19 pandemic: Effects on nationalism and anti-immigrant sentiment. Social Psychological and Personality Science. https://doi.org/10.1177/ 1948550620978023

Huang, J. Y., Sedlovskaya, A., Ackerman, J. M., \& Bargh, J. A. (2011). Immunizing against prejudice: Effects of disease protection on attitudes toward out-groups. Psychological Science, 22, 1550-1556

Ji, T., Tybur, J. M., \& van Vugt, M. (2019). Generalized or origin-specific out-group prejudice?: The role of temporary and chronic pathogen-avoidance motivation in intergroup relations. Evolutionary Psychology, 17(1), 1474704919826851

Kasahara, M. (2011). Immune system: evolutionary pressure of infectious agents. eLS 2001; Jan 1-9. https://doi.org/https://doi.org/10.1002/9780470015902.a0001131.pub.

Kim, H. S., Sherman, D. K., \& Updegraff, J. A. (2016). Fear of Ebola: The influence of collectivism on xenophobic threat responses. Psychological Science, 27, 935-944

Miller, S. L., \& Maner, J. K. (2011). Sick body, vigilant mind: The biological immune system activates the behavioral immune system. Psychological Science, 22(12), 1467-1471

Murray, D. R., \& Schaller, M. (2016). The behavioral immune system: Implications for social cognition, social interaction, and social influence. In: Olson, J. M., Zanna, M. P., (eds), Advances in experimental social psychology, Academic Press.

Ryan, S., Oaten, M., Stevenson, R. J., \& Case, T. I. (2012). Facial disfigurement is treated like an infectious disease. Evolution and Human Behavior, 33(6), 639-646

Schaller, M., \& Park, J. H. (2011). The behavioral immune system (and why it matters). Current Directions in Psychological Science, 20(2), 99-103

Publisher's Note Springer Nature remains neutral with regard to jurisdictional claims in published maps and institutional affiliations. 